\title{
Selberg Formulae for Gaussian integers
}

by

\author{
G. L. Oorren (Broadway, N. S. W., Australia)
}

Introduction. A Gaussian integer is a complex number $a+i b$ in which $a$ and $b$ are ordinaxy integers; a Gaussian prime $e$ is a Gaussian integer with $|\varrho|>1$ which is divisible only by $\varepsilon$ or $\varepsilon \varrho$ where $\varepsilon$ is $1,-1, i$ or $-i$ ( $\varepsilon \varrho$ is called an associate of $\varrho$ ). In Hardy and Wright [5] it is shown that the only Gaussian primes are $1+i$, and its associates, real primes of the form $4 n+3$ and theix associates and the factors $a+i b$ of real primes of the form $4 n+1$. Chulanorskii [1] (see also [4]) obtained the following expression for the number of Gaussian primes $\varrho$ in an expanding domain $D$ in terms of the number of Gaussian integers $\nu$ in $D$ and the radius $R$ of $D$ :

$$
\sum_{Q \in, D} 1=\frac{2}{\pi \log R} \sum_{i \in D} 1+0\left(\frac{R^{2}}{\log R \sqrt[3]{\log \log R}}\right)
$$

as $R \rightarrow \infty$. The form of the domain $D$ has been discussed by the author in an earlier paper [2]. Chulanovskii's result was obtained using elementary methods only. Kubilius [6] had previously obtained a better result (for homothetically expanding domains) using complex variable methods.

In this paper, we introduce simplified mothods for finding a number of results akin to the celebrated Selberg Formula, which is the basis of all elementary proofs of the Prime Number Theorem and extensions of it. See, for example, Erdös [3] and Wirsing [8]. Unlike Chulanorskii, we tixst find such a formula for the Gaussian integers in a disc and then apply this to the more general problem of an arbitrary domain. The former may be obtained as a direct anclogue of any of the methoda ased in the case of real integers; we tase a method of Smith-White [7] in which only simple manipulations of the Mobius function $\mu$ and its associated inversion formulae are ased.

Notation. Unless stated otherwise, the words "integers" and "primes" in the following shall refer to Gaussian integers and Gaussian primes.

Lower case Greek letters (except $\pi$ and $\xi$ ) always denote integers, with $\varrho$ reserved for primes and $\varepsilon$ for the integers $1,-1, i$ or $-i ; \xi$ is any complex number; $\theta$ is a real constant. 
Differing slightly from Chulanorskii, we shall understand $D$ to be a domain in the complex plane, of radius

$$
R=\sup _{\xi \in D}|\xi|
$$

such that the boundary consists of a bounded number of simple closed rectifiable curves, of total length $l$ and such that the ratio $l / R$ remains bounded. Without loss of generality, we shall further assume $D$ to be symmetric with respect to rotation through $90^{\circ}$; that is, if $\xi \in D$ then also $\varepsilon \xi \in D$.

By $D / v(v \neq 0)$, we shall mean the domain defined by: $\xi \in D / v$ if and only if $\xi v \in D$. Then $D / v$ is also symmetric, and has radius $R /|v|$ and boundary of length $l /|p|$. The domains $D$ and $D / v$ are geometrically similar. We shall denote by $\partial(D)$ the boundary of $D$ and by $\mathscr{A}(D)$ the area of $D$.

Preliminary results. We assume the result

$$
\sum_{|x|<R} 1=\pi R^{2}+O(R)
$$

from which

$$
\sum_{x \in \mathcal{D}} 1=O\left(R^{2}\right)
$$

Define the domain $D_{r}$ by

$$
D_{r}=\left\{\xi:\left|\xi^{\prime}-\xi^{\prime}\right|<r \text { for some } \xi^{\prime} \epsilon \partial(D)\right\}
$$

Then we show that

$$
\sum_{x \in D_{r}} 1 \leqslant B r(l+r)
$$

for some positive constant $B$.

Suppose that $\partial(D)$ consists of a single closed curve. Let $m$ satisfy $0<m \leqslant l$ and divide $\partial(D)$ into $[l / m]+1$ sections, each having length at most $m$. If $\xi \in D_{r}$, there is some $\xi^{\prime} \in \partial(D)$ for which $\left|\xi-\xi^{\prime}\right|<r$. Hence $D_{r}$ is contained in the union of circles of radius $r+m$ with centres at the division points of $\partial(D)$ and so there is a positive constant $B$ so that

$$
\sum_{x \in D_{r}} 1 \leqslant\left(\left[\frac{l}{m}\right]+1\right) \frac{B}{4}(r+m)^{2}
$$

If $l \leqslant r$, we take $m=l$, while if $l>r$, we take $m=r$, in each case obtaining (2). If $\partial(D)$ consists of a bounded number of closed curves, we simply add the above results together for the separate pieces.

It follows from (2) that

$$
\sum_{x \in D \mid w} 1=\frac{1}{|\nu|^{2}} \sum_{x \in D} 1+o\left(\frac{R}{|\nu|}\right)
$$

where $1 \leqslant|\nu| \leqslant R$. For clearly

$$
\left|\mathscr{A}(D)-\sum_{x \in D} 1\right| \leqslant \sum_{x \in D_{\sqrt{2}}} 1=O(l+\sqrt{2})
$$

so that

$$
\mathscr{A}(D)=\sum_{x \in D} 1+O(t+1)
$$

Then

$$
\mathscr{A}\left(\frac{D}{\nu}\right)=\sum_{x \in D \mid \nu} 1+O\left(\frac{l}{|\nu|}+1\right)
$$

while also

i.

$$
\mathscr{A}\left(\frac{D}{v}\right)=\frac{1}{|\nu|^{2}} \mathscr{A}(D)
$$

These lead to

$$
\sum_{x \in D / \nu} 1=\frac{1}{|\nu|^{2}} \sum_{x \in D} 1+o\left(\frac{l}{|\nu|}+1\right)
$$

since $|p| \geqslant 1$, which is a stronger result than (3). Fowever, the equation (3), which wo obtain by putting $l=O(R)$ and requiring $|y| \leqslant R$, is more useful for our purposes.

The function corresponding to the ordinary Möbius function $\mu$ is the function $q$ defined for all $a \neq 0$ by

$$
q(\alpha)= \begin{cases}1, & \text { if } a=\varepsilon, \\ 0, & \text { if } v^{2} \mid a \text { for some } v,|v|>1, \\ (-1)^{m}, & \text { if } a=\varrho_{1} \varrho_{2} \ldots \varrho_{m}, \text { where no two of the primes } \\ & \varrho_{1}, \varrho_{2}, \ldots, \varrho_{m} \text { are associates }\end{cases}
$$

Wo may readily prove, as for real integers, that

$$
\begin{gathered}
\sum_{a \mid x} q(\alpha)= \begin{cases}4, & \text { if } \quad x=\varepsilon \\
0, & \text { otherwise, }\end{cases} \\
\sum_{a \mid x} q(\alpha) \log |\alpha|= \begin{cases}-4 \log |\varrho|, & \text { if } x=\varepsilon \varrho^{a} \text { where } a \text { is } a \\
0, & \text { otherwise. }\end{cases}
\end{gathered}
$$

Define

$A(\nu)=\left\{\begin{array}{l}\log |\varrho|, \quad \text { if } \quad \nu=6 \varrho^{a} \text { where } a \text { is a positive integer, } \\ 0, \quad \text { otherwise. }\end{array}\right.$ 


$$
\begin{gathered}
\Lambda(v)=-\frac{1}{4} \sum_{\alpha \mid \nu} g(\alpha) \log |\alpha|, \\
\log |v|=\frac{1}{4} \sum_{\alpha \mid v} \Lambda(\alpha), \\
\sum_{|v|<R} \Lambda(v)=O\left\langle R^{2}\right) .
\end{gathered}
$$

The following transformations of sums are used extensively in the sequel. For convenience of notation, we now specify that henceforth the origin is always excluded from our summations.

$$
\begin{aligned}
& \sum_{|\alpha \nu|<R} f(\alpha, \nu)=\sum_{|\alpha|<R} \sum_{|p|<R|| \alpha \mid} f(\alpha, v)=\sum_{|p|<R} \sum_{|\alpha|<R /|\nu|} f(\alpha, v) \\
& =\sum_{|x|<R} \sum_{\alpha \mid x} f\left(\alpha, \frac{x}{\alpha}\right)=\sum_{|x|<R} \sum_{v \mid x} f\left(\frac{x}{v}, v\right), \\
& \sum_{\alpha, \in D} f(\alpha, v)=\sum_{|\alpha|<R} \sum_{v \in D \mid \alpha} f(\alpha, v)=\sum_{|p|<R} \sum_{a \in D \mid v} f(\alpha, v) \\
& =\sum_{x \in D} \sum_{\alpha \mid x} f\left(\alpha, \frac{x}{\alpha}\right)=\sum_{x \in D} \sum_{\nu \mid x} f\left(\frac{x}{\nu}, \nu\right) .
\end{aligned}
$$

We make considerable use of the following inversion formula:

$$
G(R)=\sum_{|p|<R} F\left(\frac{R}{|v|}\right) \quad \text { if and only if } \quad 16 F(R)=\sum_{|p|<R} q(v) G\left(\frac{R}{|\nu|}\right) .
$$

Proof. Suppose $G(R)=\sum_{|p|<R} F\left(\frac{R}{|p|}\right)$. Then

$$
\begin{aligned}
& \sum_{|\nu|<R} q(v) G\left(\frac{R}{|\nu|}\right)=\sum_{|v|<R} q(v) \sum_{|\alpha|<R /|\nu|} F\left(\frac{R}{|\alpha v|}\right)=\sum_{|\alpha v|<R} q(v) F\left(\frac{R}{|\alpha \nu|}\right) \\
& =\sum_{|x|<R} \sum_{y \mid x} q(v) F\left(\frac{R}{|x|}\right)=\sum_{|x|<R} F\left(\frac{R}{|x|}\right) \sum_{y \mid x} q(y) \\
& =4 \sum_{\substack{x \\
x=s}} F\left(\frac{R}{|x|}\right)=16 F(R)
\end{aligned}
$$

using (4). The converse is similarly proved.

If $F$ and $G$ are two functions satisfying this formula, we shall call them a Möbius Pair and denote this by $\left\{W^{\prime}, G\right\}$, an ordered pair.

Let the functions $N, \nabla, S$ and $L$ be defined for $R \geqslant 1$ by

According to $(1)$ and $(7)$,

$$
\begin{gathered}
N(R)=\sum_{|p|<R} 1, \quad N(1)=0 \\
V(R)=\sum_{|v|<R} \frac{R^{2}}{|p|^{2}}, \quad V(1)=0 ; \\
S(R)=\sum_{|v|<R} A(v), \quad S(1)=0 ; \\
L(R)=4 \sum_{|v|<R} \log |\eta|, \quad L(1)=0 .
\end{gathered}
$$

$$
N(R)=\pi R^{2}+O(R), \quad S(R)=O\left(R^{2}\right)
$$

We see that $\{1, N\}$ and $\left\{R^{2}, V\right\}$ are Möbius Pairs. Using (6),

$$
\begin{aligned}
& \sum_{|\alpha|<R} S\left(\frac{R}{|a|}\right)=\sum_{|\alpha|<R} \sum_{|p|<R /|\alpha|} \Lambda(\nu)=\sum_{|\alpha| \mid<R} \Lambda(\nu) \\
& =\sum_{|x|<R} \sum_{v \mid x} \Lambda(v)=4 \sum_{|x|<R} \log |x|=L(R),
\end{aligned}
$$

so $\{S, L\}$ is also a Möbius Pair.

Applying the inversion formula, we have

$$
\begin{aligned}
16 & =\sum_{|\nu|<R} q\langle\nu\rangle \cdot N\left(\frac{R}{|\nu|}\right), \\
16 R^{2} & =\sum_{|\nu|<R} q(\nu) V\left(\frac{R}{|\nu|}\right) .
\end{aligned}
$$

Riemann-Stieltjes integrals may be used to simplify the evaluation and estimation of sums, since a partigl summation is then replaced by an integration by parts. Integrators such as $N, S$ and $Q$, defined for $R \geqslant 1$ by

$$
Q(R)=\sum_{|\alpha|<Z 2} q(\alpha), \quad Q(\mathcal{1})=0,
$$

are all step functions, continnous from the left at thejr points of discontj.nuity. A change of dummy variable from $u$ to $v$ will always be via the. substitution $v=R / u$. Thus,

$$
\begin{aligned}
\frac{1}{4} L(R) & =\sum_{|v|<R} \log |p|=\int_{1}^{R} \log u d N(u)=[N(u) \log u]_{1}^{n}-\int_{1}^{R} \frac{N(u)}{u} d u \\
& =\pi R^{2} \log R+O(R \log R)-\int_{1}^{R} \frac{\pi u^{2}+O(u)}{u} d u \\
& =\pi R^{2} \log R-\frac{\pi}{2} \pi R^{2}+O(R \log R)
\end{aligned}
$$


so that $L(R)=4 \pi R^{2} \log R-2 \pi R^{2}+O(R \log R)$.

Likewise, we obtain

$$
\sum_{|\eta|<R} \frac{1}{|\nu|^{a}}=\int_{1}^{R} \frac{d N(u)}{u^{a}}=\left\{\begin{array}{lc}
\frac{2 \pi}{2-a} R^{2-a}+O\left(R^{1-a}\right), & a<1, \\
2 \pi R+O(\log R), & a=1, \\
\frac{2 \pi}{2-a} R^{2-a}+O(1), & 1<a<2, \\
2 \pi \log R+O+O\left(\frac{1}{R}\right), & a=2, \\
O(1), & a>2 .
\end{array}\right.
$$

In particular,

1.2)

$$
V(R)=2 \pi R^{2} \log R+O R^{2}+O(R) .
$$

Writing (8) as

$$
16=\int_{1}^{R} N\left(\frac{R}{u}\right) d Q(u)
$$

we have

$$
16=\int_{1}^{R}\left(\pi \frac{R^{2}}{u^{2}}+O\left(\frac{R}{u}\right)\right) d Q(u)=\pi R^{2} \int_{1}^{R} \frac{d Q(u)}{u^{2}}+O\left(R \int_{1}^{R} \frac{d N(u)}{u}\right)
$$

so that, by (11),

$$
\int_{1}^{R} \frac{d Q(u)}{u^{2}}=\sum_{|\alpha|<R} \frac{q(\alpha)}{|\alpha|^{2}}=O(\mathcal{I})
$$

Since $\{S, L\}$ is a Möbius Pair,

$$
\begin{aligned}
I(R) & =\int_{1}^{R} S\left(\frac{R}{u}\right) d N(u)=\left[S\left(\frac{R}{u}\right) N(u)\right]_{1}^{R}-\int_{1}^{R} N(u) d S\left(\frac{R}{u}\right) \\
& =\int_{1}^{R} N\left(\frac{R}{v}\right) d S(v)=\pi R^{2} \int_{1}^{R} \frac{d S(v)}{v^{2}}+O\left(R \int_{1}^{R} \frac{d S(v)}{v}\right)
\end{aligned}
$$

and since

$$
\int_{1}^{R} \frac{d S(v)}{v}=\sum_{|v|<R} \frac{\Lambda(v)}{|v|}=O(R)
$$

we obtain, substituting from (10),

$$
\int_{1}^{n} \frac{d S(v)}{v^{2}}=\sum_{|v|<R} \frac{\Lambda(v)}{|v|^{2}}=4 \log R+O(1) .
$$

The Selberg Formula for a dise. For any Möbins Pair $\{F, Q\}$, we have

$$
\begin{aligned}
16 W^{\prime}(R) \log R & =\sum_{|v|<R} q(v) Q\left(\frac{R}{|v|}\right) \log R \\
& =\sum_{|v|<R} q(v) G\left(\frac{R}{|v|}\right) \log \frac{R}{|v|}+\sum_{|\eta|<R} q(v) Q\left(\frac{R}{|v|}\right) \log |v| .
\end{aligned}
$$

But

$$
\begin{aligned}
\sum_{|\nu|<R} g(\nu) G\left(\frac{R}{|\nu|}\right) \log |\nu| & =\sum_{|\nu|<R} q(\nu) \log |\nu| \sum_{|\alpha|<R /|\nu|} F\left(\frac{R}{|\alpha \nu|}\right) \\
& =\sum_{|\alpha \nu|<R} q(\nu) \log |\nu| F\left(\frac{R}{|\alpha \nu|}\right) \\
& =\sum_{|x|<R} F\left(\frac{R}{|x|}\right) \sum_{\nu \mid x} g(\nu) \log |\nu|=-4 \sum_{|x|<R} F\left(\frac{R}{|x|}\right) A(x),
\end{aligned}
$$

by (5), so

(16) $\quad 16 \Pi(R) \log R+4 \sum_{|\times|<R} \Lambda(x) F\left(\frac{R}{|x|}\right)=\sum_{|\nu|<R} q(\nu) G\left(\frac{R}{|p|}\right) \log \frac{R}{|\nu|}$.

In a particular case where $G(R)=O(R \log R)$, we would have $G(R) \log R=O(R \sqrt{R})$ and

$$
\sum_{|p|<Z} q(v) G\left(\frac{R}{|v|}\right) \log \frac{R}{|\nu|}=O\left(R^{3 / 2} \sum_{|p|<R} \frac{1}{|\nu|^{3 / 2}}\right)=O\left(R^{2}\right),
$$

by (11), and in this case

$$
4 W^{\prime}(R) \log R+\sum_{|x|<Z} A(x) H\left(\frac{R}{|x|}\right)=O\left(R^{2}\right) .
$$

Now consider in enjunetion the Möbius Pairs $\{1, N\},\{S, L\}$ and $\left\{R^{2}, V\right\}$. The "smallest possible" linear combination of $N, L$ and $V$ is the function $\theta_{0}=x-2 V+2\left(1+\frac{O}{\pi}\right) N$; in fact $G_{0}(R)=O(R \log R)$. $\left\{F_{0}, G_{0}\right\}$, where $F_{0}(R)=S(R)-2 R^{2}+2\left(1+\frac{C}{\pi}\right)$, will be a Möbius Pair and (17) may be applied. 
Substituting $F_{0}$ for $F$ in (17), we get

$4 S(R) \log R-8 R^{2} \log R+8\left(1+\frac{C}{\pi}\right) \log R+\sum_{|x|<R} \Lambda(x) S\left(\frac{R}{|x|}\right)-$

$$
-2 R^{2} \sum_{|x|<R} \frac{A(x)}{|x|^{2}}+2\left(1+\frac{O}{\pi}\right) \sum_{|x|<R} \Lambda(x)=O\left(R^{2}\right) .
$$

Using (15) and simplifying, we get

$$
\log R \sum_{|v|<R} \Lambda(v)+\frac{1}{4} \sum_{|x|)<R} \Lambda(x) \Lambda(v)=4 R^{2} \log R+O\left(R^{2}\right),
$$

which is the required Selberg Formula for the integers in the disc $|\xi|<R$. Substituting $\{S, L\}$ for $\{F, G\}$ in (16) and using (18), we obtain

$$
\sum_{|v|<R} q(v) \log \frac{R}{|v|} \sum_{|x|<R /|v|} \log |x|=16 R^{2} \log R+O\left(R^{2}\right)
$$

It is the analogue in $D$ of this result that is the basis of our method for finding a Selberg Formula for the integers in $D$.

Finally in this section, we prove that

$$
\sum_{|p|<R} \frac{q(v)}{|v|^{2}} \log ^{2} \frac{R}{|p|}=\frac{16}{\pi} \log R+O(1)
$$

From (9) and (12),

$$
\begin{aligned}
16 R^{2} & =\sum_{|v|<R} q(\nu) V\left(\frac{R}{|\nu|}\right) \\
& =2 \pi R^{2} \sum_{|\nu|<R} \frac{q(\nu)}{|\nu|^{2}} \log \frac{R}{|\nu|}+C R^{2} \sum_{|v|<R} \frac{q(v)}{|\nu|^{2}}+O\left(R \sum_{|\nu|<R} \frac{1}{|\nu|}\right)
\end{aligned}
$$

so, by (11) and (13),

$$
\sum_{|\nu|<R} \frac{q(\nu)}{|\nu|^{2}} \log \frac{R}{|\nu|}=O(1)
$$

Substituting (10) into (19):

$16 R^{2} \log R+O\left(R^{2}\right)$

$$
\begin{aligned}
& =\pi R^{2} \sum_{|\nu|<R} \frac{q(\nu)}{|\nu|^{2}} \log ^{2} \frac{R}{|\nu|}-\frac{1}{2} \pi R^{2} \sum_{|p|<R} \frac{q(\nu)}{|\nu|^{2}} \log \frac{R}{|\nu|}+O\left(R \sum_{|\nu|<R} \frac{1}{|\nu|} \log ^{2} \frac{R}{|\nu|}\right) \\
& =\pi R^{2} \sum_{|p|<H} \frac{q(\nu)}{|\nu|^{2}} \log ^{2} \frac{R}{|\nu|}+O\left(R^{2}\right)+O\left(R^{3 / 2} \sum_{|\nu|<R} \frac{1}{|\nu|^{3 / 2}}\right)
\end{aligned}
$$

and (20) follows, using (11) and (21).
The Sellierg Formula for $D$. Set

On the one hand,

$$
I^{\prime}=\sum_{|\nu|<R} q(\nu) \log \frac{R}{|\nu|} \sum_{x \in D \mid \nu} \log |\varkappa| .
$$

$$
\begin{aligned}
& T=\sum_{|p|<R} q(\nu) \log \frac{R}{|\nu|} \sum_{\psi \in \mathcal{L} D \mid v}\left(\log \frac{R}{|\nu|}+\left(\log |x|-\log \frac{R}{|\nu|}\right)\right) \\
& \sum_{|p|<X i} q(\nu) \log ^{2} \frac{R}{|\nu|} \sum_{x \in D \mid \nu} 1+D
\end{aligned}
$$

where

$$
E=\sum_{|\nu|<R} q(\nu) \log \frac{R}{|\nu|} \sum_{x \in D / \nu} \log \frac{|\chi \nu|}{R} .
$$

By (3) and (20),

$$
\begin{aligned}
& \Gamma=\sum_{|p|<R} q(v) \log ^{2} \frac{R}{|\nu|}\left(\frac{1}{|v|^{2}} \sum_{x \in D} 1+o\left(\frac{R}{|v|}\right)\right)+E \\
& =\sum_{|p|<R} \frac{q(\nu)}{|\nu|^{2}} \log ^{2} \frac{R}{|\nu|} \sum_{x \in D} 1+O\left(R^{3 / 2} \sum_{|\varphi|<R} \frac{1}{|\nu|^{3 / 2}}\right)+E \\
& =\frac{16}{\pi} \log R \sum_{x \in D} 1+O\left(R^{2}\right)+D \text {. }
\end{aligned}
$$

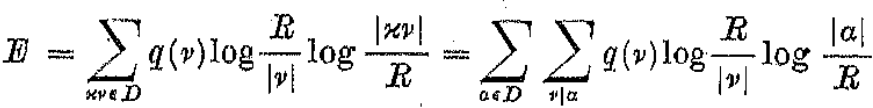

$$
\begin{aligned}
& =\sum_{\alpha \in D} \log \frac{|\alpha|}{R} \sum_{p \mid a} q(\nu)(\log R-\log |v|) \\
& =\log R \sum_{\alpha \in D} \log \frac{|\alpha|}{R} \sum_{p \mid \alpha} q(v)-\sum_{\alpha \in D} \log \frac{|\alpha|}{R} \sum_{p \mid \alpha} q(v) \log |p| \\
& =-16 \log ^{2} R-4 \sum_{i \in \mathbb{D}} \Lambda(\alpha) \log \frac{R}{|\alpha|}=O\left(\log ^{2} R\right)+O\left(\sum_{|\alpha|<R} \Lambda(\alpha) \log \frac{R}{|\alpha|}\right),
\end{aligned}
$$

using (4) and (5). We have assumed here that $1 \epsilon D$, but elearly this is not necessary. Now,

$$
\begin{aligned}
\sum_{|\alpha|<R} A(\alpha) \log \frac{R}{|\alpha|} & =\int_{1}^{\pi} \log \frac{R}{u} d S(u)=\left[S(u) \log \frac{R}{u}\right]_{1}^{R}+\int_{1}^{R} \frac{S(u)}{u} d u \\
& =O\left(\int_{1}^{R} u d u\right)=O\left(R^{2}\right)
\end{aligned}
$$


so $B=O\left(R^{2}\right)$ and

$$
\Gamma=\frac{16}{\pi} \log R \sum_{x \in D} 1+O\left(R^{2}\right)
$$

On the other hand, notice that, by $(6)$,

$$
4 \sum_{x \in D} \log |x|=\sum_{x \in D} \sum_{v \mid x} \Lambda(v)=\sum_{\mu, \in \mathcal{D}} \Lambda(v)=\sum_{|v|<R} \Lambda(v) \sum_{\mu \in D / p} 1 .
$$

Then,

$$
\begin{aligned}
& 4 \Gamma=\sum_{|\nu|<R} q(\nu) \log \frac{R}{|\nu|} \sum_{|x|<R /|v|} A(x) \sum_{\mu \in D \mid x p} 1=\sum_{|x|<R} q(v) \log \frac{R}{|\nu|} \Lambda(x) \sum_{\mu \in D \mid x \nu} 1 \\
& =\sum_{|x|<R} \Lambda(x) \sum_{|p|<R /|x|} q(\nu) \log \frac{R}{|\nu|} \sum_{\mu \in D \mid x v} x=\sum_{|x|<R} \Lambda(x) \sum_{\mu, \epsilon, D / \varkappa} q(\nu) \log \frac{R}{|\nu|} \\
& =\log R \sum_{|x|<R} \Lambda(x) \sum_{\alpha \in D \mid x} \sum_{v \mid \alpha} q(v)-\sum_{|x|<R} \Lambda(x) \sum_{\alpha \in D \mid x} \sum_{p \mid \alpha} q(v) \log |v| \\
& =4 \log R \sum_{\substack{\alpha, x \\
\alpha \in D \\
\alpha \neq \varepsilon}} \Lambda(x)+4 \sum_{|x|<R} \Lambda(x) \sum_{\substack{\alpha \in D / x \\
\alpha \neq \delta}} \Lambda(\alpha) \\
& =16 \log R \sum_{x \in D} \Lambda(x)+4 \sum_{u x \in D} \Lambda(x) \Lambda(\alpha),
\end{aligned}
$$

using (4) and (5). Thus

$$
\text { (23) } \quad \Gamma=4 \log R \sum_{x \in D} \Lambda(x)+\sum_{a x \in D} \Lambda(x) \Lambda(\alpha) \text {. }
$$

From (22) and (23),

$$
\text { (24) } \quad \log R \sum_{x \in D} \Lambda(x)+\frac{1}{4} \sum_{a x \in D} \Lambda(a) \Lambda(x)=\frac{4}{\pi} \log R \sum_{x \in D} 1+O\left(R^{2}\right)
$$

which is the required Selberg Formula for the integers in $D$.

Further formulae. We next obtain some alternative forms and extensions of (24).

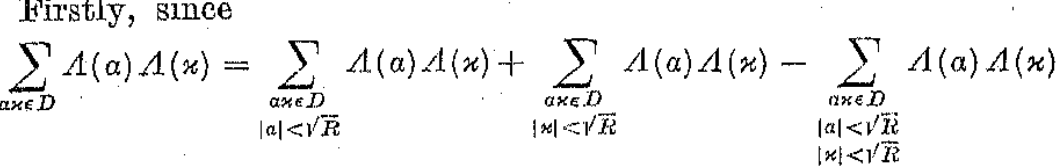

$$
\begin{aligned}
& =2 \sum_{|\mathrm{a}|<V \bar{R}} \Lambda(\alpha) \sum_{x \in D / \alpha} \Lambda(x)+O\left(R^{2}\right),
\end{aligned}
$$

(24) may be given equivalently as

(25) $\log R \sum_{x \in D} A(x)+\frac{1}{2} \sum_{|\alpha|<\gamma / \bar{R}} A(\alpha) \sum_{x \in D \mid \alpha} A(x)=\frac{4}{\pi} \log R \sum_{x \in D} 1+O\left(R^{2}\right)$.
Secondly, we show that

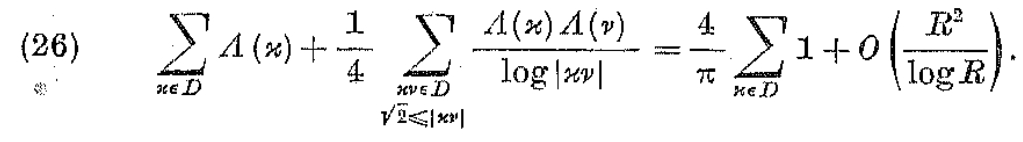

Introduce as an integrator the function $S_{1}$, defjned for $R \geqslant 1$ by

$$
S_{x}(R)=\sum_{|x p|<R} \Lambda(x) \Lambda(v), \quad S_{1}(1)=0
$$

By $(24), S_{1}(R)=O\left(R^{2} \log R\right)$. Then

$$
\begin{aligned}
\sum_{\sqrt{2}<|x v|<R} \frac{\Lambda(x) \Lambda(\nu)}{\log |x v|} \log \frac{R}{|x v|} & =\int_{\sqrt{2}}^{R} \frac{\log (R / u)}{\log u} d S_{1}(u) \\
& =\left[S_{1}(u) \frac{\log (R / u)}{\log u}\right]_{\sqrt{2}}^{R}+\log R \int_{\sqrt{2}}^{R} \frac{S_{1}(u)}{u \log ^{2} u} d u \\
& =O(\log R)+O\left(\log R \int_{\sqrt{2}}^{R} \frac{u}{\log u} d u\right)=O\left(R^{2}\right)
\end{aligned}
$$

and so

$$
\begin{aligned}
& \sum_{x \in D} \Lambda(x)+\frac{1}{4} \sum_{\substack{x \in \in \in D \\
\sqrt{2} \in|x p|}} \frac{\Lambda(x) \Lambda(p)}{\log |x v|}
\end{aligned}
$$

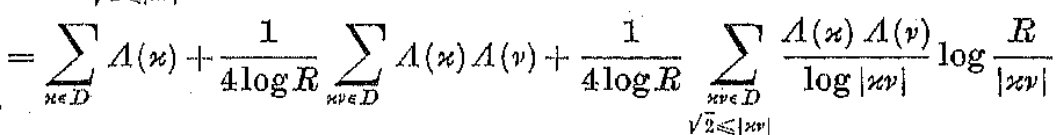

$$
\begin{aligned}
& =\frac{4}{\pi} \sum_{x \in D} 1+O\left(\frac{R^{2}}{\log R}\right)+O\left(\frac{1}{\log R} \sum_{\sqrt{2} \ll|x v|<R} \frac{\Lambda(x) \Lambda(v)}{\log |x p|} \log \frac{R}{|x v|}\right) \\
& =\frac{4}{\pi} \sum_{x \in D} x+O\left(\frac{R^{2}}{\log R}\right)
\end{aligned}
$$

by (24). It will be convenient below to replace $O\left(R^{2} / \log R\right)$ in $(26)$ by $O\left(R^{2} / \log 2 R\right)$.

Our next result is:

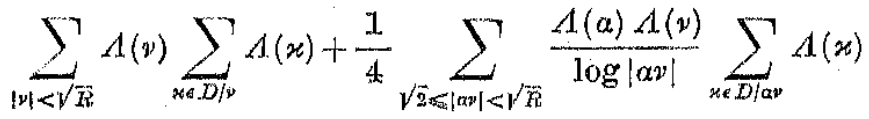

$$
\begin{aligned}
& =\frac{8}{\pi} \log R \sum_{\nu \in D} 1+O\left(R^{2} \log \log R\right) .
\end{aligned}
$$


This is a special case of the following: Let $h$ be a real-valued function defined on the positive real numbers, and suppose that the function $H$, given by

$$
H(R)=\sum_{|x|<R} h(|x|)(R>1), \quad H(1)=0,
$$

satisfies the condition $H(R)=O\left(R^{2}\right)$. Then

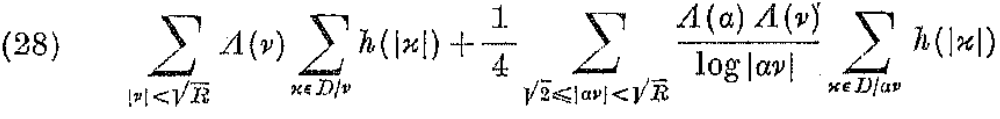

$$
\begin{aligned}
& =\frac{4}{\pi} \sum_{\sqrt{R} \in|x|<R} \frac{h(|x|)}{|x|^{2}} \sum_{\nu \in D} 1+O\left(R^{2} \log \log R\right) .
\end{aligned}
$$

To derive (27) from (28), we simply note that it is ralid to put $h(|x|)=A(x)$ and we use (1.5).'

The proof of (28) requires the estimates

$$
\sum_{\sqrt{2} \leqslant|x y|<\sqrt{\bar{R}}} \frac{\Lambda(\varkappa) \Lambda(v)}{\log |\varkappa v|}=O(R)
$$

easily proved with $S_{1}$ as integrator,

$$
\sum_{|x|<R} \frac{h(|x|)}{|x|}=O(R)
$$

easily proved with $H$ as integrator, and

proved as follows:

$$
\sum_{|x|<\Omega} \frac{h(|x|)}{|x|^{2} \log (2 R /|x|)}=O(\log \log R)
$$

$$
\begin{aligned}
\sum_{|x|<R} \frac{h(|x|)}{|x|^{2} \log (2 R /|x|)} & =\int_{1}^{R} \frac{d H(u)}{u^{2} \log (2 R / u)} \\
& =\left[\frac{H(u)}{u^{2} \log (2 R / u)}\right]_{1}^{R}+\int_{1}^{R} H(u) \frac{2 \log (2 R / u)-1}{u^{3} \log ^{2}(2 R / u)} d u \\
& =O(1)+O\left(\int_{1}^{R} \frac{d u}{u \log (2 R / u)}\right)+O\left(\int_{1}^{R} \frac{d u}{u \log ^{2}(2 R / u)}\right) \\
& =O(1)+O\left(\int_{1}^{R} \frac{d v}{v \log 2 v}\right)+O\left(\int_{1}^{R} \frac{d v}{v \log ^{2} 2 v}\right) \\
& =O(\log \log R) .
\end{aligned}
$$

Then,

$$
\begin{aligned}
& \sum_{|\eta|<\sqrt{R}} \Lambda(v) \sum_{x \in D \mid v} h(|x|)+\frac{1}{4} \sum_{\sqrt{2} \leqslant|\alpha v|<V \bar{R}} \frac{A(\alpha) A(\nu)}{\log |\alpha \nu|} \sum_{x \in D \mid \alpha \nu} h(|x|)
\end{aligned}
$$

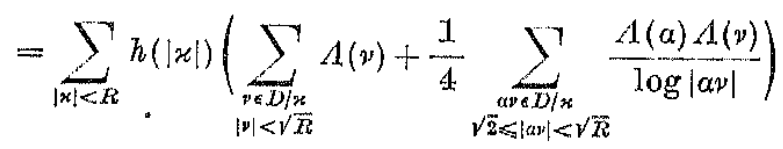

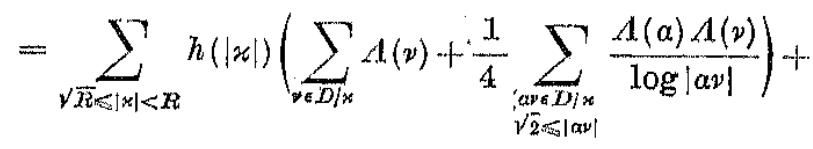

$$
\begin{aligned}
& +o\left(\sum_{|x|<\sqrt{R}} h(|x|)\left(\sum_{|v|<\sqrt{R}} A(v)+\sum_{v \sqrt{2} \leq|\alpha|<\mid<\sqrt{R}} \frac{A(a) A(v)}{\log |\alpha v|}\right)\right) \\
& =\sum_{\sqrt{\bar{R} \leqslant|x|<R}} h(|x|)\left(\frac{4}{\pi} \sum_{\nu \in D / x} 1+O\left(\frac{R^{2}}{|x|^{2} \log (2 R /|x|)}\right)\right)+O\left(R^{2}\right) \\
& =\frac{4}{\pi} \sum_{V|\bar{x} \leqslant| x \mid<R} h(|x|)\left(\frac{1}{|x|^{2}} \sum_{p \in D} 1+O\left(\frac{R}{|x|}\right)\right)+ \\
& +O\left(R^{2} \sum_{|x|<R} \frac{h(|x|)}{|x|^{2} \log (2 R /|x|)}\right)+O\left(R^{2}\right) \\
& =\frac{4}{\pi} \sum_{\sqrt{R} \leqslant|x|<R} \frac{h(|x|)}{|x|^{2}} \sum_{x \in D} 1+O\left(R^{2} \log \log R\right\rangle,
\end{aligned}
$$

in which we have also used (26) and (3).

Subtracting a quarter of (27) from (25) gives us a formula of Selbergtype which is basic to Chulanovskii's method:

(29)

$$
\begin{aligned}
& \log R \sum_{x \in D} A(x)+\frac{1}{4} \sum_{|| v \mid<V / \mathbb{Z}} A(v) \sum_{x \in D \mid v} A(x)- \\
& -\frac{1}{16} \sum_{\sqrt{2} \leqslant \leqslant|\alpha \nu|<\sqrt{R}} \frac{\Lambda(\alpha) \alpha 1(\nu)}{\log |\alpha \nu|} \sum_{x \in D / \alpha v} A(x) \\
& =\frac{2}{\pi} \log R \sum_{p \in D} x+\dot{O}\left(R^{2} \log \log R\right) .
\end{aligned}
$$

5 - Acta Arithmetica $\mathrm{xxvi}$. 
Finally, in this section, we show that

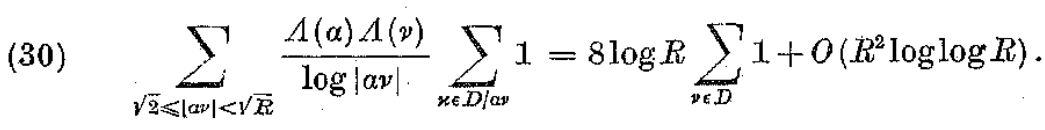

Putting $h(|x|)=1$ in (28) and rearranging, we have

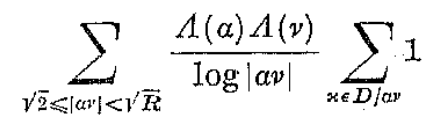

$$
\begin{aligned}
& =\frac{16}{\pi} \sum_{\sqrt{R} \leqslant|x|<R} \frac{1}{\left.|x|\right|^{2}} \sum_{\nu \in D} 1-4 \sum_{|\nu|<\sqrt{R}} \Lambda(v) \sum_{x \in D \mid \nu} 1+O\left(R^{2} \log \log R\right)
\end{aligned}
$$

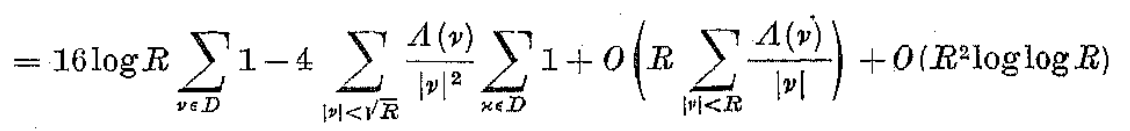

$$
\begin{aligned}
& =8 \log R \sum_{v \in D} 1+O\left(R^{2} \log \log R\right),
\end{aligned}
$$

using (11), (3), (15) and (14).

The remainder term $\mathscr{G}(D)$. Chulanorskii estimated the number of primes in $D$ by obtaining a satisfactory estimate for the remainder term $\mathscr{G}(D)$, defined by

$$
\sum_{p \in D} A(p)=\frac{2}{\pi} \sum_{p \in D} 1+\mathscr{G}(D)
$$

We conclude by deriving a fundamental inequality satisfied by $\mathscr{G}(D)$. This is a consequence of substituting from (31) into (29).

Under (31), we have

$$
\begin{aligned}
& \sum_{|p|<1 \bar{R}} \Lambda(v) \sum_{x \in D / v} \Lambda(x) \\
& =\sum_{|v|<\nu^{\prime} \bar{R}} \Lambda(v)\left(\frac{2}{\pi} \sum_{x \in D \mid p}^{\top} 1+\mathscr{G}\left(\frac{D}{\nu}\right)\right) \\
& =\frac{2}{\pi} \sum_{|p|<\sqrt{R}} \frac{A(\nu)}{|\nu|^{2}} \sum_{x \in D} 1+o\left(R \sum_{|p|<R} \frac{A(\nu)}{|\nu|}\right)+\sum_{|v|<V / \mathbb{R}} A(\nu) \mathscr{G}\left(\frac{D}{\nu}\right) \\
& =\frac{4}{\pi} \log R \sum_{v \in D} 1+\sum_{|v|<\backslash / \bar{R}} \Lambda(v) \mathscr{G}\left(\frac{D}{\nu}\right)+O\left(R^{2}\right),
\end{aligned}
$$

by (3), (14) and (15); and

$$
\begin{aligned}
& \sum_{\sqrt{2} \leqslant|\alpha \nu|<\sqrt{R}} \frac{A(\alpha) A(\nu)}{\log |\alpha \nu|} \sum_{x \in D \backslash a w^{\prime}} A(x)
\end{aligned}
$$

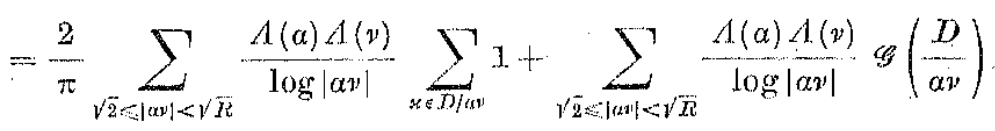

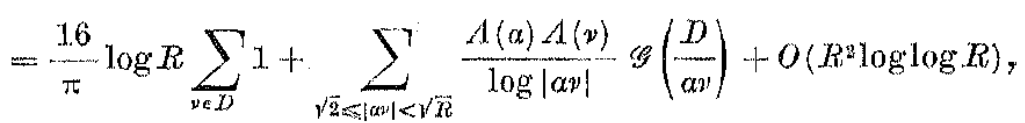

by (30). Hence, carrying out the substitition,

$$
\begin{array}{r}
\mathscr{G}(D) \log R+\frac{1}{4} \sum_{|\nu|<\sqrt{R}} \Lambda(\nu) \mathscr{G}\left(\frac{D}{\nu}\right)-\frac{1}{16} \sum_{\sqrt{2} \leqslant|\alpha v|<\gamma / \bar{R}} \frac{A(\alpha) A(\nu)}{\log |\alpha \nu|} \mathscr{G}\left(\frac{D}{\alpha \nu}\right) \\
=O\left(R^{2} \log \log R\right) .
\end{array}
$$

The terms in $\sum_{v \in D} 1$ cancel out, accounting for the method of formation of (29). The required inequality is

$$
\begin{aligned}
|\mathscr{G}(D)| \leqslant & \frac{1}{4 \log R} \sum_{|\nu|<\sqrt{R}} \Lambda(\nu)\left|\mathscr{G}\left(\frac{D}{\nu}\right)\right|+ \\
& +\frac{1}{16 \log R} \sum_{\sqrt{2} \leqslant|\alpha \nu|<\sqrt{R}} \frac{\Lambda(\alpha) \Lambda(\nu)}{\log |\alpha \nu|}\left|\mathscr{G}\left(\frac{D}{\alpha \nu}\right)\right|+o\left(\frac{R^{2} \log \log R}{\log R}\right) .
\end{aligned}
$$

Acknowledgements. The author would like to acknowledge the assistance given him by Professor W. B. Smith-White and Dr. J. M. Mack, of the University of Sydney.

\section{References}

[I] I. V. Chulanovakii, An elementary proof of the law of distribution of primes of a Ganssian fiold (in Russian), Vestaik LGU 13 (1956), pp, 43-62.

[2] G. "L. Cohen, Boundary conditions for expanding domains, Aota Arith. 26. (1975), pp. 213-216.

[3] P. Erd ö, On a new method in olementary number theory whioh leads to an elementary proof of the prime number theorem, Proc. Nat. Aoad. Sci. U.S.A. 35 (1949), pp. $374-384$.

[4.] A. O. Golfond and Yu. V. Linnik, Hlementary Methods in Analytio Number Theory, Allen and Unwin 1965.

[5] G. H. Hardy and E. M. Wright, An Introduction to the Theory of Numbers, fonrth edition, Oxford 1962. 
6] I. P. Kubilius, The distribution of primes of a Ganssian field in sectors and contours (in Russian), Učen. Zap. LGU, ser. matem., 19 (1950), pp. 40-52.

[7] W. B. Smith-White, The elementary proof of the prime number theorem, Uni. versity of Sydney, Australia (1967). Uupublished.

[8] E. Wirsing, Elementare Beweise des Primzahlsatzes mit Restglied, II, J. Reine Angew. Math. 215 (1964), pp. 1-18.

N. S. W. INSTITUTE OF THCHNOLOGY

Broadway, Austratia

\section{Absolutely continuous distribution functions of additive functions $f(p)=(\log p)^{-a}, a>0$}

by

\section{G. Joghsr. BABr** (Calcutta)}

Introduction. The following question was raised by Erdös in a private communication to the author. Suppose $f$ is a real-valued additive arithmetic function such that, for all primes $p$,

$$
f(p)=(\log p)^{-a}
$$

for some $a>0$. For which values of $a>0$ does $f$ have an absolutely continuous distribution? In the same communication Erdös pointed out that $f$ has absolutely continuous distribution for $a=1$.

In this paper it is shown that $f$ has an absolutely continuous distribution if $0<a<2$.

Notations and definilions. An arithmetic function $f$ is said to be additive if

$$
f(m n)=f(m)+f(n)
$$

whenever $(m, n)=1 . f$ is called strongly additive if, in addition, $f$ satisfies $f\left(p^{\hbar k}\right)=f(p)$ for all primes $p$ and for all positive integers $k$.

A real-valued arithmetic function $h$ is said to have a distribution if there exists a distribution function $\theta$ such that the density of $\{m \geqslant 1$ : $h(m) \leqslant c\}$ exists and equals $G(c)$, whenever $o$ is a continuity point of $G$. Throughout this paper we let $p$ denote a prime number.

\section{The result.}

Tworim. Let $f$ be a real valued additive arithmetio function satisfying for all primes $p$,

$$
f(p)=(\log p)^{-a}
$$

for some $0<a<2$. Then $f$ has an absolutely continuous distribution.

Proof. That $f$ has a distribution follows from Erdös-Wintner theorem (see [2]). From the results of [1] it follows that the distribution of $f$ is absolutely continuous if and only if the distribution of the corresponding

* The author is currently a Visiting Member at the TIPR, Bombay. 